\title{
Factors Associated With Discussions of Human Immunodeficiency Virus Pre-Exposure Prophylaxis in Men Who Have Sex With Men
}

\author{
Barley R. Halton, MD, ${ }^{1}$ Jill N. T. Roberts, MD, ${ }^{1}$ G. Dodd Denton, MD, MPH ${ }^{1,2}$ \\ ${ }^{1}$ The University of Queensland Faculty of Medicine, Ochsner Clinical School, New Orleans, LA 2 Department of Internal Medicine, Ochsner \\ Clinic Foundation, New Orleans, LA
}

Background: Pre-exposure prophylaxis (PrEP) with emtricitabine/tenofovir disoproxil fumarate (Truvada) is highly effective at preventing human immunodeficiency virus (HIV) transmission in high-risk populations, including in men who have sex with men (MSM). In 2019, the US Preventive Services Task Force released an A recommendation to offer PrEP to persons at high risk of HIV acquisition. Despite the demonstrated efficacy of PrEP, areas with high HIV incidence, such as Louisiana, have historically had low PrEP prescription rates. The objective of this study was to determine the factors associated with whether providers in the Ochsner Health System (OHS) discussed PrEP with HIV-negative MSM patients.

Methods: Investigators extracted electronic medical record data on all HIV-negative MSM patients who had at least one outpatient visit at OHS between July 1, 2012 and July 1, 2016 and manually reviewed a random sample of 115 charts.

Results: Subjects were predominantly Caucasian (75.7\%) with a mean age of 37.6 years. A PrEP discussion was documented for 34 (29.6\%) patients. Multivariate modeling showed that having a PrEP discussion was associated with 3 factors: being assigned to a primary care provider known to specialize in MSM care (odds ratio [OR] 5.05, 95\% confidence interval [CI] 1.81-14.10; $P=0.002$ ), having a documented history (positive or negative) of sexually transmitted infection vs no documentation (OR 5.41, 95\% $\mathrm{Cl} 1.80$ 16.23; $P=0.003)$, and having documentation of condom use (consistent or inconsistent) vs no documentation (OR $3.32,95 \% \mathrm{Cl}$ 1.27-8.74; $P=0.015)$.

Conclusion: Despite evidence that PrEP significantly reduces sexual transmission of HIV in MSM, PrEP discussions with MSM across OHS were undesirably low. Additional resources need to be aimed at increasing PrEP uptake and should focus on providing skillsbased training and education in PrEP and MSM care to healthcare providers. With increased knowledge of and familiarity with PrEP prescribing guidelines, more providers will be better equipped to identify at-risk patients and to discuss prevention options such as PrEP.

Keywords: Anti-HIV agents, emtricitabine, tenofovir disoproxil fumarate drug combination, HIV, pre-exposure prophylaxis, sexual and gender minorities

Address correspondence to G. Dodd Denton MD, MPH, Department of Internal Medicine, Ochsner Clinic Foundation, 1401 Jefferson Highway, New Orleans, LA 70121. Tel: (504) 842-4747. Email: gdenton@ochsner.org

\section{INTRODUCTION}

Pre-exposure prophylaxis (PrEP) with once-daily emtricitabine/tenofovir disoproxil fumarate (Truvada) is highly effective at preventing human immunodeficiency virus (HIV) transmission in high-risk populations, particularly men who have sex with men (MSM). ${ }^{1}$ The iPrEx trial conducted by Grant et al and published in 2010 was the first study to evaluate the effectiveness of PrEP in MSM and demonstrated that when taken daily, emtricitabine/tenofovir disoproxil fumarate reduced the risk of contracting HIV by more than $90 \%$ in some individuals. ${ }^{1,2}$ Two additional studies of emtricitabine/tenofovir disoproxil fumarate con- ducted in the United States and published in 2013 reported zero seroconversions among participants. ${ }^{3,4}$ Following the success of the iPrEx study, the US Food and Drug Administration approved emtricitabine/tenofovir disoproxil fumarate for use in July 2012, ${ }^{5}$ and the World Health Organization modified its guidelines to recommend PrEP as an additional prevention choice for people at substantial risk of HIV infection. ${ }^{6}$

The Centers for Disease Control and Prevention (CDC) published clinical practice guidelines in 2014 about PrEP use in the United States and updated the guidelines in $2017 .{ }^{7}$ The guidelines state that once-daily oral treatment with 
the fixed-dose combination of tenofovir disoproxil fumarate $300 \mathrm{mg}$ and emtricitabine $200 \mathrm{mg}$ is safe and effective in reducing the risk of HIV acquisition in adults. The CDC recommends PrEP as one prevention option for sexually active adult MSM at substantial risk of contracting HIV. ${ }^{7}$

In June 2019, the US Preventive Services Task Force (USPSTF) published an A recommendation that PrEP should be offered to persons at high risk of HIV acquisition. ${ }^{8}$ An A recommendation means that the service has a strong net benefit and that practitioners should provide it. Among the persons at high risk of HIV acquisition listed in this recommendation are sexually active HIV-negative MSM who have one or more of these characteristics: an HIV-positive sex partner, inconsistent condom use, and/or a diagnosis of a sexually transmitted infection (STI) within 6 months.

In 2017, the southern United States accounted for more than half of new HIV diagnoses, and Louisiana had the second highest HIV incidence rate in the United States (22.1 new HIV diagnoses per 100,000 people), primarily among African American MSM. ${ }^{9}$ In 2015, the Baton Rouge and New Orleans metropolitan statistical areas ranked second and third in the nation for new HIV case rates (32.0 and 31.9 per 100,000 people, respectively). ${ }^{10}$

Despite the high incidence rate of HIV in Louisiana, the adoption of PrEP for HIV prevention in the state has been slow. From 2012-2015, Louisiana accounted for only $0.9 \%$ of PrEP prescriptions in the United States, ${ }^{11}$ despite ranking second in the nation for HIV case rates (24.2 per 100,000 people) in $2015 .^{10}$

As the largest healthcare system in Louisiana, Ochsner Health System (OHS) provides primary care to a large cohort of MSM patients, and the Epic electronic medical record (EMR) facilitates large-scale data collection from multiple clinical sites. The OHS structure of multiple centers connected by one EMR allows for easy tracking of the care of individuals with different socioeconomic and demographic characteristics. Analysis of patient-physician encounters can provide insight into HIV prevention efforts across OHS and Louisiana.

The aim of this study was to determine the patient and provider factors associated with discussions about PrEP between HIV-negative MSM patients and their physicians.

\section{METHODS}

We extracted data from the EMR for all male patients who had at least one outpatient visit at OHS between July 1, 2012 and July 1, 2016 and who were identified as having male sexual partners in the sexual activity portion of their social history $(n=2,230)$. Data downloaded from the EMR included whether the patient had solely male or both male and female sexual partners, race, age, health insurance, primary healthcare provider (including specialty and location of the practice), results of all HIV testing, and whether at any point during the study period the patient received a prescription for emtricitabine/tenofovir disoproxil fumarate. We also extracted results for 6 HIV-associated diagnostic codes (International Statistical Classification of Diseases [ICD], Ninth Revision [ICD-9] codes 493, 878 and 42 and ICD-10 codes V08, Z21, and B20) for this cohort and eliminated 339 patients with an existing HIV diagnosis, leaving a cohort of 1,891 MSM patients without HIV. Every patient was assigned a unique study identification number to ensure patient privacy, and these identifiers were used for all data analyses.

The Ochsner Clinic Foundation Institutional Review Board approved this study on August 4, 2016, as a minimal risk project meeting the requirements for waiver of consent under 45CFR 46.116(d). The study was reapproved under continuing review on July 26, 2017.

Investigators manually reviewed a random sample of charts. To determine sample size, we assumed that an alpha of 0.05 , a power of 0.80 , and a between-group difference of $15 \%$ increased the likelihood that a conversation about PrEP took place when HIV testing was performed. Because we found errors in the documentation of sexual orientation in the EMR, we doubled the sample size, and a random sample of 360 patients was chosen for in-depth chart review. Each investigator reviewed 120 charts. Reviews were limited to the 4-year time frame of the study.

To assess whether PrEP should have been discussed with the patient during a primary care visit and to identify the patient factors associated with this conversation, we developed a chart review tool. Each investigator was assigned 5 random charts to review with the tool. Investigators met to discuss difficulties encountered using the tool and altered the tool by consensus. This process was repeated 5 times, each time using a new random sample of charts and an updated tool until all investigators agreed upon the tool. In addition to providing space for free-text answers, the final chart review tool included the following questions:

1. Who was the primary care provider listed on patient's EMR?

2. Was the patient sexually active during the study period? (yes/no/undocumented)

3. Are you confident the patient is MSM? (yes/no/lack of enough documentation)

4. Was the patient tested for HIV during the study period? (yes/no)

5. Was HIV PrEP or postexposure prophylaxis discussed with the patient during the study period? (yes/no)

6. Does the patient consistently use condoms? (yes/no/ undocumented)

7. Has the patient ever had an STI? (yes [list of types]/no/undocumented)

8. Was the patient tested for an STI other than HIV during the study period? (yes [list of types]/no/undocumented)

9. Does the patient have a history of nonprescription drug use, including marijuana? (yes/no/undocumented)

10. Does the patient have a history of intravenous drug use? (yes/no/undocumented)

11. Does the patient have any of the following contraindications to PrEP? (chronic kidney disease/osteopenia/ osteoporosis/none known)

12. Is the patient transgender? (yes, female to male/yes, male to female/no)

If the patient was prescribed PrEP, the name, specialty, and location of the prescribing provider were recorded.

Once the chart review tool was finalized, each investigator extracted data from the EMR for each of his/her randomly assigned charts. If the investigator was not confident that the patient was MSM, this finding was documented, and further chart review was aborted. 


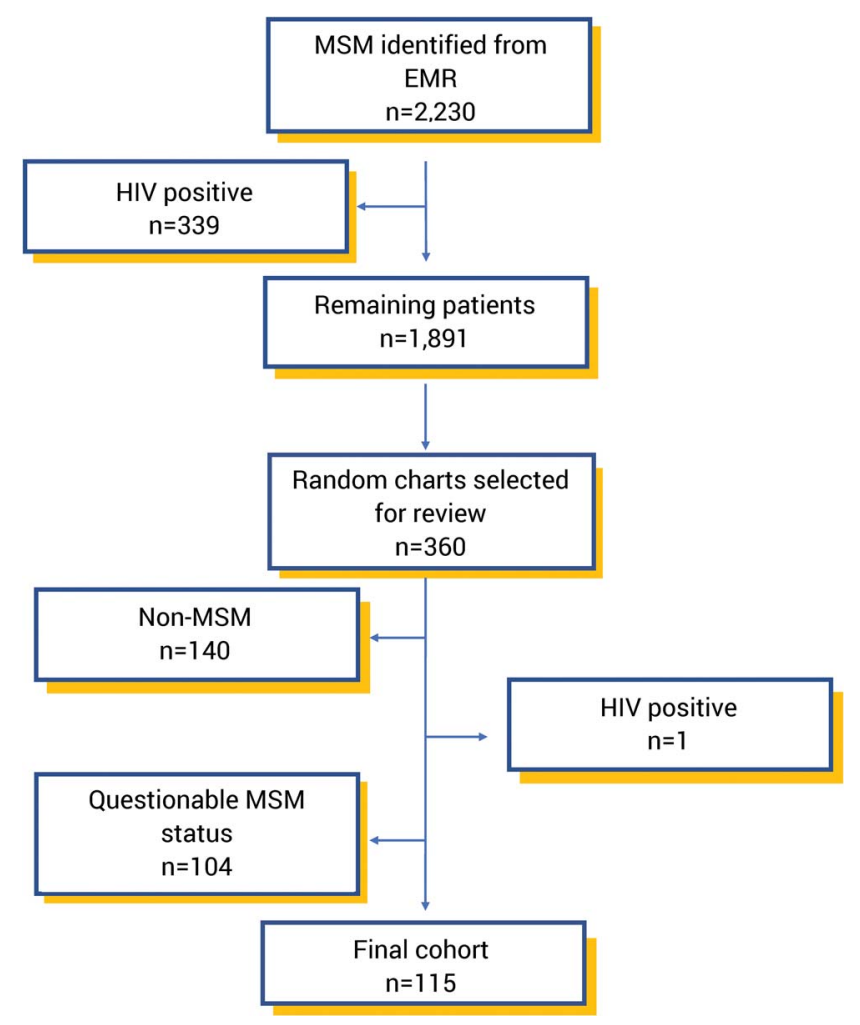

Figure. Derivation of the study cohort.

To measure agreement among providers, percent agreement was calculated. Each investigator reviewed 10 randomly chosen charts from the other 2 investigators $(20$ charts in total). Percent agreement across 6 questions (questions 2-6 and 9) was $87.6 \%$.

Chi-square test was used to compare all variables with the principal outcome of whether the patient and physician discussed PrEP. Logistic regression analysis was used to evaluate the interactions among variables. All data were analyzed using Stata statistical software v.10 (StataCorp, LLC).

\section{RESULTS}

EMR documentation of sexual orientation was errorprone. Manual chart review revealed that 140 patients did not actually have male sexual partners, and 104 patients lacked sufficient documentation to determine the sex of their sexual partners. In addition to these 244 patients, an additional patient was excluded because he had been diagnosed with HIV infection prior to the study period. The final cohort included 115 patients (Figure): 111 patients who were clearly MSM and HIV negative and 4 male-to-female transgender patients who had male sexual partners and were HIV negative. Nine of the 115 patients reported both female and male sexual partners.

The majority of patients were Caucasian (75.7\%), and the mean age of all patients was 37.6 years (range, 19-76 years). PrEP was discussed with 34 patients $(29.6 \%)$ during the study period, and 20 patients (17.4\%) were prescribed PrEP (Table 1).

During the study period $35.3 \%$ (6/17) of African American patients had a PrEP discussion vs $28.7 \%$ (25/87) of Caucasian patients $(P=\mathrm{NS})$. Seventy-four patients $(64.3 \%)$ were
Table 1. Demographic Characteristics of the Study Cohort $(n=115)$

\begin{tabular}{lc}
\hline Variable & Number of Patients (\%) \\
\hline Age $\leq 40$ years & $74(64.3)$ \\
Race/ethnicity & \\
$\quad$ Caucasian & $87(75.7)$ \\
$\quad$ African American & $17(14.8)$ \\
$\quad$ Hispanic/Latino & $2(1.7)$ \\
$\quad$ Other & $9(7.8)$ \\
Saw MSM specialist & $36(31.3)$ \\
Sexually active & $108(93.9)$ \\
Condom use & \\
$\quad$ Consistent & $9(7.8)$ \\
$\quad$ Inconsistent & $33(28.7)$ \\
$\quad$ Undocumented & $73(63.5)$ \\
Prior history of STIs & $20(17.4)$ \\
HIV testing & $72(62.6)$ \\
STI testing (other than HIV) & $73(63.5)$ \\
Documented PrEP discussion & $34(29.6)$ \\
Prescribed PrEP & $20(17.4)$ \\
\hline
\end{tabular}

${ }^{a}$ Asian, Pacific Islander, other, or declined.

' Including gonorrhea, chlamydia, syphilis, herpes simplex virus type 1 , herpes simplex virus type 2, hepatitis B.

HIV, human immunodeficiency virus; MSM, men who have sex with men; PrEP, pre-exposure prophylaxis (emtricitabine/tenofovir disoproxil fumarate [Truvada]); STI, sexually transmitted infection.

age 40 or younger, and $36.5 \%(27 / 74)$ of these patients had a PrEP discussion during the study period vs $17.1 \%(7 / 41)$ of those $>40$ years $(P=0.029)$.

Twenty patients (17.4\%) had a clearly documented STI in their medical record, and 6 patients $(5.2 \%)$ had clear documentation of no prior history of STI. Having any STI documentation in the chart (positive or negative) was associated with a PrEP discussion during the study period $(57.7 \%$ vs $21.4 \%, P<0.001$ ) (Table 2).

Condom use was documented for 42 patients (36.5\%). Having any documentation of condom use (consistent or inconsistent) was associated with a PrEP discussion during the study period ( $50.0 \%$ vs $17.8 \%, P<0.001)$.

Seventy-two patients (62.6\%) were tested for HIV during the study period. Testing for HIV during the study period was associated with having a PrEP discussion $(47.0 \%$ vs $0 \%, P<0.001)$. Seventy-three patients $(63.5 \%)$ were tested for STIs other than HIV during the study period. Testing for STIs other than HIV was also associated with having a PrEP discussion $(42.0 \%$ vs $7.0 \%, P<0.001)$.

Two of the 52 primary care physicians who provided care for this study population were known to care for large cohorts of MSM patients. These 2 physicians cared for 36 patients in the study group (10 and 26 , respectively). No other primary care provider had more than 4 patients in the study group. Patients assigned to these 2 physicians had PrEP discussions more frequently than patients assigned to other providers $(47.2 \%$ vs $21.5 \%, P=0.005)$. These 2 physicians also prescribed PrEP more frequently than the other 
Halton, $B R$

Table 2. Univariate Analysis of Patient and Provider Variables Associated With Having a PrEP Discussion During a Primary Care Visit

\begin{tabular}{|c|c|c|c|}
\hline Variable & $\begin{array}{l}\text { PrEP Conversations } \\
\text { With Variable } \\
\text { Present, \% }\end{array}$ & $\begin{array}{l}\text { PrEP Conversations } \\
\text { With Variable } \\
\text { Absent, \% }\end{array}$ & $P$ Value \\
\hline Documentation of STI history (positive or negative) & 57.7 & 21.4 & $<0.001$ \\
\hline Documentation of condom use (consistent or inconsistent) & 50.0 & 17.8 & $<0.001$ \\
\hline HIV testing & 47.0 & 0.0 & $<0.001$ \\
\hline STI testing (other than HIV) & 42.0 & 7.0 & $<0.001$ \\
\hline Saw MSM specialist & 47.2 & 21.5 & 0.005 \\
\hline Sexually active & 31.5 & 0.0 & 0.077 \\
\hline
\end{tabular}

HIV, human immunodeficiency virus; MSM, men who have sex with men; PrEP, pre-exposure prophylaxis (emtricitabine/tenofovir disoproxil fumarate [Truvada]); STI, sexually transmitted infection.

providers $(25.0 \%$ vs $13.9 \%, P=\mathrm{NS})$, although the difference did not reach statistical significance. In addition, these 2 physicians provided more HIV testing $(83.3 \%$ vs $53.1 \%$, $P=0.002$ ) and tested more frequently for STIs other than HIV (88.9\% vs $51.9 \%, P<0.001)$ than other providers. However, being assigned to 1 of the 2 primary care providers with large numbers of MSM patients was not associated with documentation of STI history or documentation of condom use.

The majority of patients $(93.9 \%)$ were sexually active during the study period. These patients had a PrEP discussion more frequently than those who were not sexually active (31.5\% vs $0 \% ; P=0.077$ ), although the difference was not statistically significant.

Only 10 patients had a clearly documented history of nonprescription drug use, including marijuana. One had a history of intravenous drug use. These numbers were too small to analyze.

In multivariate logistic regression modeling (Table 3), three variables had significant associations with having a PrEP discussion: being assigned to 1 of the 2 primary care physicians

Table 3. Multiple Logistic Regression Analysis of Patient and Provider Variables Associated With Having a PrEP Discussion During a Primary Care Visit

\begin{tabular}{lccc}
\hline Variable & $\begin{array}{c}\text { Odds } \\
\text { Ratio }\end{array}$ & $\begin{array}{c}\boldsymbol{P} \text { Value } \\
\text { Confidence } \\
\text { Interval }\end{array}$ \\
\hline $\begin{array}{l}\text { Saw MSM specialist } \\
\begin{array}{l}\text { Documentation of STI history } \\
\text { (positive or negative) }\end{array}\end{array}$ & 5.05 & 0.002 & $1.81-14.10$ \\
$\begin{array}{l}\text { Documentation of condom use } \\
\text { (consistent or inconsistent) }\end{array}$ & 3.32 & 0.003 & $1.80-16.23$ \\
$\begin{array}{l}\text { Age } \leq 40 \text { years } \\
\text { African American race }\end{array}$ & 1.74 & 0.340 & $0.55-5.48$ \\
\hline
\end{tabular}

Note: Absence of HIV testing and no sexual activity during the study period both perfectly predicted no PrEP discussion and were forced out of the model.

MSM, men who have sex with men; PrEP, pre-exposure prophylaxis (emtricitabine/tenofovir disoproxil fumarate [Truvada]); STI, sexually transmitted infection. known to care for large numbers of MSM (odds ratio [OR] 5.05, 95\% confidence interval [Cl] 1.81-14.10; $P=0.002$ ); having documentation of STI history (positive or negative) (OR 5.41, 95\% Cl 1.80-16.23; $P=0.003$ ); and having documentation of condom use (consistent or inconsistent) (OR $3.32,95 \% \mathrm{Cl} 1.27-8.74 ; P=0.015)$.

\section{DISCUSSION}

Despite evidence that PrEP can reduce sexual transmission of HIV in MSM by more than $90 \%,{ }^{1}$ PrEP discussion and prescription rates for MSM across OHS were undesirably low (29.6\% and $17.4 \%$, respectively). The most significant factor influencing whether a discussion about PrEP took place between patients and their providers was being assigned to 1 of 2 physicians who provide primary care for large numbers of MSM. Assignment is not a random process, and these providers may have been sought by MSM seeking PrEP. As with sex and race, patients may look for primary care physicians of the same sexual orientation. Concordance across social factors has been associated with better and more open physician-patient communication. ${ }^{12}$

While the majority $(76 \%)$ of primary care providers are aware of PrEP and support its use as a public health intervention, prescription currently remains limited to few early adopters. ${ }^{13}$ These early adopters, represented in this study by the 2 providers with large numbers of MSM patients, have a role in decreasing barriers to PrEP implementation by communicating to colleagues their successful experiences with PrEP.

Potential barriers underlying the slow uptake of PrEP include provider concern about the efficacy and long-term safety of PrEP and perceived issues in prescribing PrEP in real-world settings, ${ }^{14}$ such as cost and adherence. Additionally, because of suboptimal HIV risk assessment, many providers struggle to identify patients who would benefit from PrEP. ${ }^{14}$ For this reason, some providers are now using innovative tools such as the PrEP score tool, developed by the CDC, that calculates a numeric score indicating an individual's risk of becoming HIV positive and whether the patient is a strong candidate for PrEP. ${ }^{15}$

Since the time period when this study was conducted, the USPSTF released its strongest recommendation that PrEP should be offered to high-risk individuals. Among the 
highest risk individuals are sexually active HIV-negative MSM who have HIV-positive partners, do not consistently use condoms, and have recently had an STI. This recommendation should push providers to increase documentation of risk factors and recommend PrEP more frequently. This recommendation should also prompt health systems to implement strategies, such as EMR decision aids, to identify patients at highest risk. ${ }^{8}$

On a statewide level, Louisiana public health officials have begun to address the high incidence rate of HIV and improve PrEP utilization by implementing programs such as the New Orleans Louisiana Pre-exposure Prophylaxis Program (NOLA PrEPP) and New Orleans Links (NOLA Links) under Project PrIDE as a means to specifically reduce new HIV infections in MSM and transgender persons. ${ }^{16}$ Louisiana receives funding for both projects from the CDC. Goals of these efforts are to increase PrEP awareness and its use, establishing it as a staple in HIV prevention. ${ }^{17}$ According to the HIV program director for the Louisiana Department of Health and Hospitals, NOLA Links will expand upon and enhance the already in place LA Links that enrolled approximately 500 people during its first 2 years. Of the 500 people initially enrolled, the majority (87\%) started receiving medical treatment. ${ }^{18}$ In December 2016, New Orleans implemented the Rapid Start Program, a relatively new initiative only available in a few cities across the country. This program provides patients with the means to get tested and learn their HIV test results within minutes, and if positive, to start antiretroviral therapy within 72 hours of diagnosis. ${ }^{19}$ If the test is negative, patients have the opportunity to discuss PrEP for HIV prevention with their provider.

\section{Limitations and Strengths}

Our study has several limitations, most attributable to the retrospective nature of the data collection. PrEP discussion may have prompted questions about STI and condom use rather than vice versa. PrEP discussion may also have prompted HIV and other STI testing. No causality can be inferred. Care provided outside OHS was not captured. Because of errors in the documentation of the sex of patients' sexual partners, investigators only included patients with clear documentation of MSM behavior. Some of the 244 eliminated patients may have been MSM. Overall, patient sexuality is poorly documented in the EMR, and these records may not adequately reflect discussions of condom use or of PrEP that did not result in a prescription. Furthermore, we had no way to verify whether every PrEP discussion was documented; consequently, many more patients may have had such discussions with their providers. This study only included MSM, the highest risk group for HIV transmission, and results cannot be extrapolated to other patient populations at risk for HIV.

While this study may be generalizable to a similar patient population, the demographics of participants in this study do not reflect the demographics of Southeast Louisiana. The greater New Orleans area population is $59.8 \%$ African American, but only $14.8 \%$ of our cohort identified as such. ${ }^{20}$

Strengths of this study include a large sample size of HIVnegative MSM, a robust EMR that enabled data collection, and good agreement among the 3 investigators in their chart reviews.
Future directions for investigation include identifying gaps in knowledge among providers regarding MSM sexual health and PrEP prescription guidelines.

\section{CONCLUSION}

This study aimed to determine the patient and provider factors associated with having a discussion about PrEP among HIV-negative MSM across a large Louisiana health system. Three factors were identified as being significant: being assigned to a primary care physician known to specialize in MSM care, having documentation of condom use (either consistent or inconsistent), and having documentation of STI history (either positive or negative).

These findings highlight the importance of establishing a trusting patient-physician relationship and honing clinical skills such as performing physical examinations and obtaining thorough patient histories that include sexual health practices. Such skills are essential to enable providers to identify the patients most at risk for acquiring HIV, whether they are MSM, transgender, or otherwise engaging in highrisk sexual practices. Additional resources now need to be aimed at increasing PrEP uptake and should focus on providing skills-based training and education in PrEP and MSM care to healthcare providers. With increased knowledge of and familiarity with PrEP prescribing guidelines, more providers, especially those practicing primary care in HIVprevalent areas such as Louisiana, will be better equipped to identify at-risk patients and to discuss prevention options such as PrEP.

\section{ACKNOWLEDGMENTS}

This research was supported in part by a $\$ 2,500$ intramural grant from the Ochsner Clinical School. Otherwise, the authors have no financial or proprietary interest in the subject matter of this article.

\section{REFERENCES}

1. Pre-Exposure Prophylaxis (PrEP). Centers for Disease Control and Prevention website. www.cdc.gov/hiv/risk/prep/. Updated November 1, 2018. Accessed May 10, 2019.

2. Grant RM, Lama JR, Anderson PL, et al. Preexposure chemoprophylaxis for HIV prevention in men who have sex with men. N Engl J Med. 2010 Dec 30;363(27):2587-2599.

3. Grohskopf LA, Chillag KL, Gvetadze R, et al. Randomized trial of clinical safety of daily oral tenofovir disoproxil fumarate among HIV-uninfected men who have sex with men in the United States. J Aquir Immune Defic Syndr. 2013 Sep;64(1):79-86. doi: 10.1097/QAI.0b013e31828ece33.

4. Hosek SG, Siberry G, Bell M, et al. The acceptability and feasibility of an HIV preexposure prophylaxis (PrEP) trial with young men who have sex with men. J Aquir Immune Defic Syndr. 2013 Apr 1;62(4):447-456. doi: $10.1097 /$ QAI.0b013e3182801081.

5. FDA approves first drug for reducing the risk of sexually acquired HIV infection. AIDS Info. U.S. Department of Health and Human Services. aidsinfo.nih.gov/news/1254/ fda-approves-first-drug-for-reducing-the-risk-of-sexuallyacquired-hiv-infection. Published July 16, 2012. Accessed February 13, 2018.

6. Consolidated guidelines on the use of antiretroviral drugs for treating and preventing HIV infection: recommendations for a public health approach. World Health Organization. 
www.who.int/hiv/pub/guidelines/arv2013/en/. Published June 2013. Accessed February 13, 2018.

7. Preexposure prophylaxis for the prevention of HIV infection in the United States - 2017 update. A clinical practice guideline. Centers for Disease Control and Prevention. www.cdc.gov/ hiv/pdf/risk/prep/cdc-hiv-prep-guidelines-2017.pdf. Accessed May 12, 2019.

8. US Preventive Services Task Force, Owens DK, Davidson KW, Krist AH, et al. Preexposure prophylaxis for the prevention of HIV infection: US Preventive Services Task Force Recommendation Statement. JAMA. 2019 Jun 11;321(22):2203-2213. doi: 10.1001/jama.2019.6390.

9. HIV in the United States by Region. Centers for Disease Control and Prevention. www.cdc.gov/hiv/statistics/overview/ geographicdistribution.html. Updated November 27, 2018. Accessed February 13, 2018.

10. 2015 STD/HIV Surveillance Report. Louisiana Department of Health Office of Public Health. http://ldh.la.gov/assets/oph/ HIVSTD/2015_STDHIV_SurveillanceReport.pdf. Accessed March 18, 2018.

11. Levin J. FTC/TDF (Truvada) for HIV pre-exposure prophylaxis (PrEP) utilization in the United States: 2012-2015. www.natap.org/2016/IAC/IAC_17.htm. Published July 2016. Accessed 13 February 13, 2018.

12. Banerjee A, Sanyal D. Dynamics of doctor-patient relationship: a cross-sectional study on concordance, trust, and patient enablement. J Family Community Med. 2012 Jan;19(1):12-19. doi: 10.4103/2230-8229.94006.

13. Petroll AE, Walsh JL, Owczarzak JL, McAuliffe TL, Bogart LM, Kelly JA. PrEP awareness, familiarity, comfort, and prescribing experience among US primary care providers and HIV specialists. AIDS Behav. 2017 May;21(5):1256-1267. doi: 10.1007/s10461-016-1625-1.

14. Krakower D, Ware N, Mitty JA, Maloney K, Mayer KH. HIV providers' perceived barriers and facilitators to implementing pre-exposure prophylaxis in care settings: a qualitative study. AIDS Behav. 2014 Sep;18(9):1712-1721. doi: 10.1007/s10461-014-0839-3.

15. PrEP'D AF. Los Angeles LGBT Center. prephere.org/. Accessed June 17, 2018.

16. Louisiana Department of Health \& Hospitals Funded Categories. Centers for Disease Control and Prevention. www.cdc.gov/hiv/pdf/research/demonstration/ executive_summaries/cdc-hiv-pride-Louisiana.pdf. Accessed March 15, 2018.

17. Project PrIDE. Centers for Disease Control and Prevention. www.cdc.gov/hiv/research/demonstration/projectpride.html. Accessed March 15, 2018.

18. Gallo A. Despite innovations in treatment, HIV rates still high, including in Baton Rouge and New Orleans. The Advocate. December 30, 2015. www.theadvocate.com/baton_rouge/ news/article_53df0406-6084-553c-baef-698becaa3ac9.html. Accessed March 15, 2018.

19. Curth K. There's a new way to fight the HIV epidemic in New Orleans. FOX8. February 4, 2017. Updated August 12, 2017 www.fox8live.com/story/34424431/theres-a-new-way-to-fightthe-hiv-epidemic-in-new-orleans. Accessed March 18, 2018.

20. QuickFacts: New Orleans city, Louisiana. United States Census Bureau. www.census.gov/quickfacts/neworleanscitylouisiana. Published July 1, 2018. Accessed May 10, 2019.

This article meets the Accreditation Council for Graduate Medical Education and the American Board of Medical Specialties Maintenance of Certification competencies for Patient Care, Medical Knowledge and Practice-Based Learning and Improvement. 